Original article

\title{
Experimental muscle reinnervation by allogeneic biomaterial illustrates restoration of interlevel relations in human body
}

\author{
Lyalya A. Musina ${ }^{1}$, Olga R. Shangina ${ }^{1}$, Rafik T. Nigmatullin ${ }^{1}$, Sagit A. Muslimov ${ }^{1}$ \\ ${ }^{1}$ Russian National Center of Eye and Plastic Surgery, Ufa, Russia
}

Received 10 April 2020, Accepted 26 May 2021

Original Text (C) Musina L.A, Shangina O.R., Nigmatullin R.T., Muslimov S.A., 2020, published in Saratov Journal of Medical Scientific Research 2020; 16 (2): 623-627.

(C) 2021, Musina L.A., Shangina O.R., Nigmatullin R.T., Muslimov S.A.

(C) 2021, Saratov Medical Journal

\begin{abstract}
:
The objective of our study was to reveal the morphofunctional interactions between allografts and the recipient nervous system from the experimental model of the mimetic musculature reinnervation.

Materials and Methods. The experiments were conducted on Chinchilla rabbits $(n=36)$. All animals were subjected to the facial nerve transection. No other manipulations were performed in the Control Group $(n=9)$. In Treatment Group 1 ( $n=12)$, an autograft fragment of the masseter muscle with a neurovascular bundle was attached to the denervated buccinator. In Treatment Group $2(\mathrm{n}=15)$, allogeneic biomaterials - specifically, Regeneration Stimulator and Vasculogenesis Stimulator, were inserted between specified muscles. The animals were removed from the experiment on days 10, 30, 60 and 180. Tissue pieces from the operation zone were analyzed using transmission electron microscopy.

Results. It was established that compensatory and restorative processes in the Control Group and Treatment Group 1 end with scarring of the boundary zone and contracture of mimetic muscles. In Treatment Group 2, we observed revascularization of mimetic musculature, as well as axonal ingrowth into the buccinator and restoration of neuromuscular synapses.

Conclusion. Allogeneic biomaterial transplantation creates adequate conditions for the restoration of the organ vascular bed and the innervation apparatus of denervated mimetic muscles. The results of our experiments could be regarded as an example of restoring the interlevel relations in the human body following the use of allogeneic biomaterials.
\end{abstract}

Keywords: muscle reinnervation, biomaterials, allotransplantation.

Cite as Musina LA, Shangina OR, Nigmatullin RT, Muslimov SA. Experimental muscle reinnervation by allogeneic biomaterial illustrates restoration of interlevel relations in human body. Saratov Medical Journal 2021; 2 (2): e0202.

Correspondence to Lyalya A. Musina, Tel.: +7(917)7750710. E-mail: morphoplant@mail.ru

\section{Introduction}

In the second half of the past century, academician V.P. Filatov proposed that tissue transplants emit specific factors (biostimulants), which perpetrate their regulatory and metabolic effects at different structural and functional organismic levels. Based on this theoretical concept, he developed a medical technology of tissue therapy and successfully implemented it in clinical practice. This was confirmed by his publications on effective treatment of a number of ophthalmic diseases, systemic lesions of connective tissue, trophic ulcers, complications of chronic infectious diseases (including cavernous forms of pulmonary tuberculosis), various types of endocrinopathies and other pathological conditions with tissue implantation [1].

Currently, local processes of replacement regeneration are well understood. At the same time, the phenomenon of systemic reactions including regulatory mechanisms of restorative morphogenesis, according to A.G. Babaeva [2], "was and remains the most problematic and least clear". In her opinion, this fact is connected with the response of the organism. As a rule, several integrative systems are involved in this response, providing interorgan coordination and correlation in the reparative process dynamics. According to some researchers, there is every reason to consider nervous, immune, endocrine and connective tissue systems components of such integrative complex [3]. Based on the foregoing, we believe that further study of the role of regulatory systems in ensuring the morphogenetic dynamics against the background of tissue transplantation is of both theoretical and practical interests. The obvious progress of recent decades in the methods of morphological studies established necessary prerequisites for solving the problems under consideration from the standpoint of the structuralfunctional and systemic-structural approaches.

Objective: to reveal the morphofunctional interactions between allografts and the recipient nervous system from the experimental model of the mimetic musculature reinnervation.

\section{Materials and methods}

The experiment was carried out on Chinchilla rabbits (weight 2,500-3,500 g). When working with animals, the international principles of the Declaration of Helsinki on the Humane Treatment of Animals (2000) and the provisions of the Federal Law of January 1, 1997, On the Protection of Animals from Cruelty were observed. In all rabbits, right 
facial nerves innervating the buccinators were transected. The skin incision was made from the auricle to the corner of the mandible. The parotid gland was dissected and moved forward, while separated facial nerve was exposed at the exit from the temporal bone. In order to exclude the possibility of facial nerve fiber penetration, its trunk was ligated with a compression silk ligature. The incision of the main trunk of the nerve was performed along the outer edge of the ligature; soft tissues were sutured layer by layer.

In the Control Group $(n=9)$, reinnervation was not performed and grafts were not used. In Treatment Group 1 $(n=12)$, after transection of the facial nerve, a myoplastic surgery was performed with the insertion of a muscle flap (from the masseter muscle) with a neurovascular bundle without use of any allogeneic biomaterials; therefore, we conducted the autotransplantation. The resulting muscle autograft retained the intraorgan vascular and nervous structures associated with the belly of the masseter muscle, and was sutured to the denervated layer of mimetic muscles. In Treatment Group $2(\mathrm{n}=15)$, an identical myoplastic surgery was performed, but a layer of dispersed forms of Alloplant $\AA$ biomaterials, Regeneration Stimulator and Vasculogenesis Stimulator, manufactured according to Technical Conditions 9398-001-04537642-2011), was inserted between the masseter autograft and the layer of mimetic muscles. Rabbits were removed from the experiment on days 10, 30, 60 and 180 after the surgery.

Small 1-2 $\mathrm{mm}$ pieces of tissue from the operated area were subjected to electron microscopic examination. The fixation and filling of the material was carried out by conventional methods [4]. Pieces of tissue were fixed in $2.5 \%$ glutaraldehyde in a cacodylate buffer $(\mathrm{pH}$ 7.2-7.4) for 2 hours with additional fixation in a $1 \%$ solution of osmium tetroxide $\left(\mathrm{OsO}_{4}\right)$ ( 1 hour in a refrigerator at a temperature of $\left.+4^{\circ} \mathrm{C}\right)$. The material was dehydrated in a battery of alcohols of increasing concentration and absolute acetone, embedded in EPON 812 resin and polymerized at $37{ }^{\circ} \mathrm{C}$ and $60{ }^{\circ} \mathrm{C}$ in a thermostat. Ultrathin sections were prepared using LKB-III 8800 ultramicrotome (Sweden). The sections were contrasted with a $2 \%$ aqueous solution of uranyl acetate and lead citrate, studied and photographed in an electron microscope JEM-1011 (JEOL Ltd., Japan) at magnification of 2500-20000.

\section{Results}

In the Control Group, morphological changes in the buccinator muscles were characteristic of the skeletal musculature denervation process. Following the dystrophic processes of muscle cells, atrophic processes were developing as well. Gradually, the muscle fibers were homogenized and disintegrated (Figure 1a). Thinning of fibers and hypertrophy of nuclei were taking place, and the pattern of cross striation was smoothed out. Synapses with nerve appendages were not detected on collapsing muscle cells.

In described area, the number of active fibroblastic cells with numerous dilated channels of the granular endoplasmic reticulum (GER) increased, indicating an increase in the collagen synthesis function of cells (Figure $1 b$ ). In place of destroyed muscle fibers, bundles of dense connective tissue grew. The latter was enlarging by the end of the experiment, and ultimately, the processes taking place in the muscle tissue were leading to the fibrosis or scarring.

In Treatment Group 1, a month later, a connective tissue regenerate was also beginning to form in the boundary zone; although, in the autogenous flap of the masseter, single regenerating nerve fibers were detected, probably, extending from the branching of the trigeminal nerve. Despite this, during all periods of the experiment, the newly formed nerve bundles near the buccinators, along with any neuromuscular synaptic junctions, were not detected. The fibers of the resected facial nerve were gradually undergoing Wallerian degeneration, whereas the fibers of the buccinator were being gradually destroyed (Figure $2 a$ ).

By the day 180, the fibers of the buccinator were mostly destroyed and atrophied without additional innervation. In the boundary zone, a dense connective tissue scar was detected, and partially preserved muscle fibers near it were exposed to pronounced contraction (Figure $2 b$ ).

In Treatment Group 2, on the day 30 after the surgery, the structure of buccinators differed from the structure of denervated muscles in animals of Treatment Group 1: the area of loose connective tissue was formed in the boundary zone between the buccinator and the masseter flap, promoting faster axonal sprouting from the bundles of trigeminal nerve to denervated buccinator muscle. In two months, and later on in the experiment, blood capillaries and separate unmyelinated neuronal axons were visible between the fibers of the regenerating muscle. In such areas, neuromuscular junctions (motor end plates) were identified in the form of synapses with their characteristic numerous contact folds, forming on the surface of the muscle fiber, with numerous synaptic vesicles (containing acetylcholine neurotransmitter) (Figure 3a). Accumulations of optically dark elongated mitochondria were visualized here as well, which could be explained by the high energy cost of the processes occurring in neuromuscular junctions. In majority of the muscle cells, clearly delineated nuclei of a rounded or somewhat elongated shape with lumps of heterochromatin along their internal karyolemma were seen. Around them, a moderate number of rounded or oval mitochondria with thin cristae, scarce short GER channels, accumulations of ribosomes and polyribosomes, and glycogen asterisks was concentrated. The transverse striation in the muscles was relatively well pronounced.

By the day 180 , the buccinator ultrastructure was approaching to normal (Figure $3 b$ ). The striation of the muscle tissue was clearly visible. Between the muscle bundles, blood vessel profiles, separate myelinated and unmyelinated axons, lying parallel along the muscle cells, were observed.

\section{Discussion}

After damage to the facial nerve as a result of breaking structural and functional relations with buccinators, muscle paralysis, muscle tissue atrophy, and mimetic muscle scarring and contracture most often develop. That was exactly the picture we observed in the Control Group of rabbits. In contrast, in Treatment Group 1, where partial autotransplantation of the masseter was performed, the processes of buccinator tissue destruction were significantly slowed down. Probably, the sewn head of the buccinator had a certain trophic effect, but this was not enough to stimulate tissue regeneration. Destructive processes in the damaged muscle intensified, and the compensatory and restorative processes ended in fibrosis and scarring of the boundary zone [5]. The formed dense connective tissue could not facilitate the sprouting of blood vessels and nerve fibers from the muscle autograft into the area of the damaged mimetic muscle: obviously, the scar prevented full regeneration. 


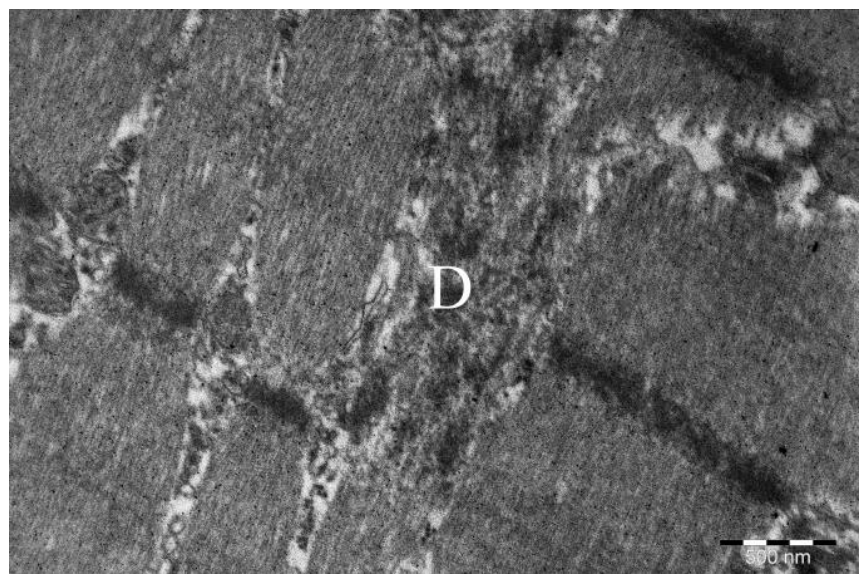

Figure 1a. Ultrastructure of denervated buccinators in control rabbits. Destruction (D) of the muscle fiber on day 30 after facial nerve resection. Magnification x20000

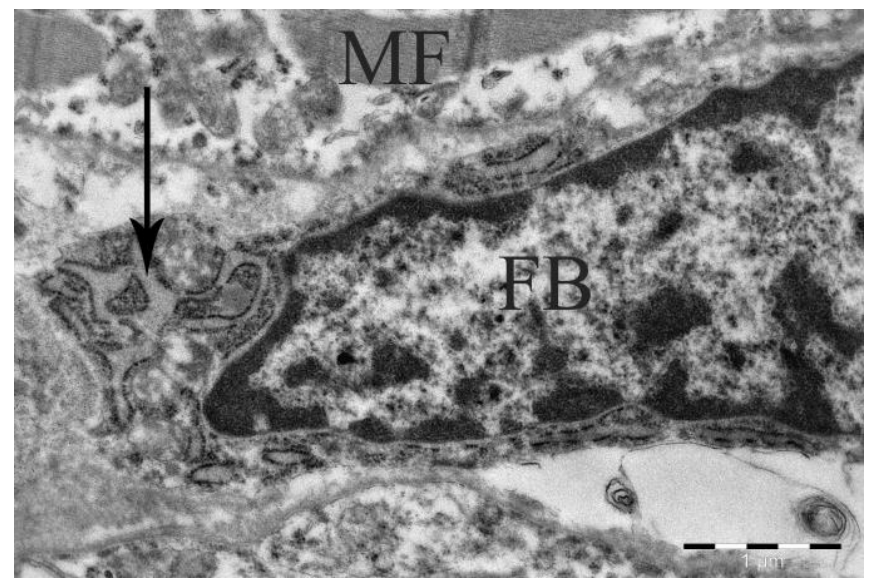

Figure 1b. Ultrastructure of denervated buccinators in control rabbits. Active fibroblast (FB) with dilated GER channels $(-)$ along the collapsing muscle fiber (MF) near the developing connective tissue scar on day 60 after facial nerve resection. Magnification x15000

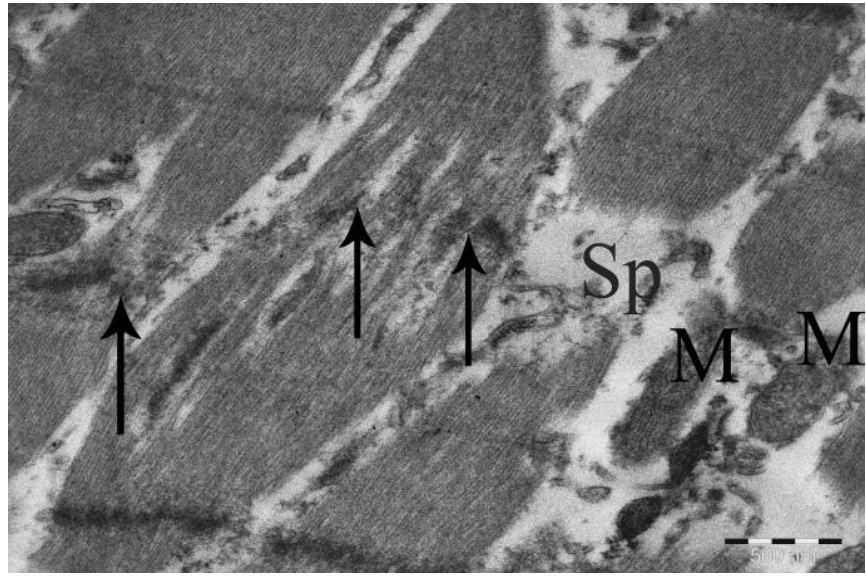

Figure 2a. Ultrastructure of denervated buccinators in rabbits of Treatment Group 1 after the surgery without use of allogeneic biomaterials. Destruction of fibers in buccinators on day 6o; destruction of mitochondria (M); absence of glycogen granules in the sarcoplasm (Sp), lysis of individual sarcomeres (-). Magnification x20000

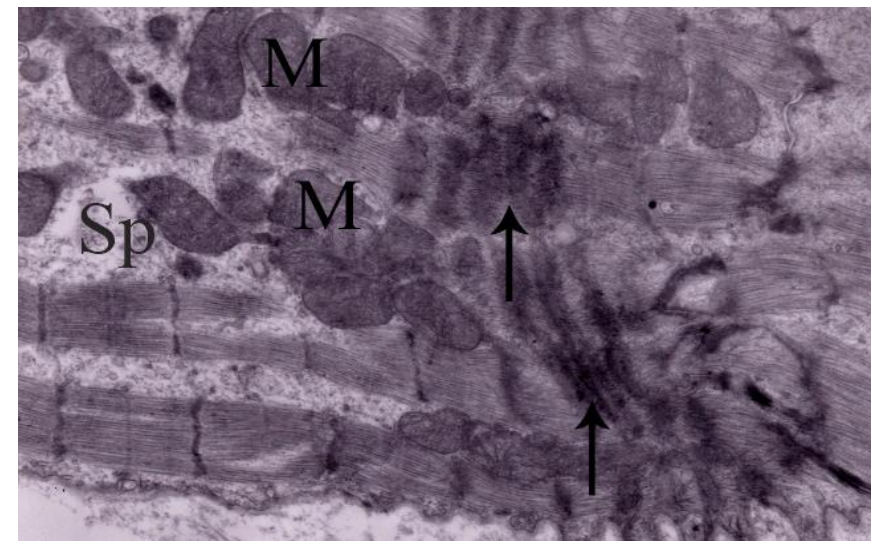

Figure 2b. Ultrastructure of denervated buccinators in rabbits of Treatment Group 1 after the surgery without use of allogeneic biomaterials. Contraction (-) of fibers in the buccinator near the connective tissue scar on day 180 after the surgery; Sp - sarcoplasm; M - mitochondria. Magnification x1500o

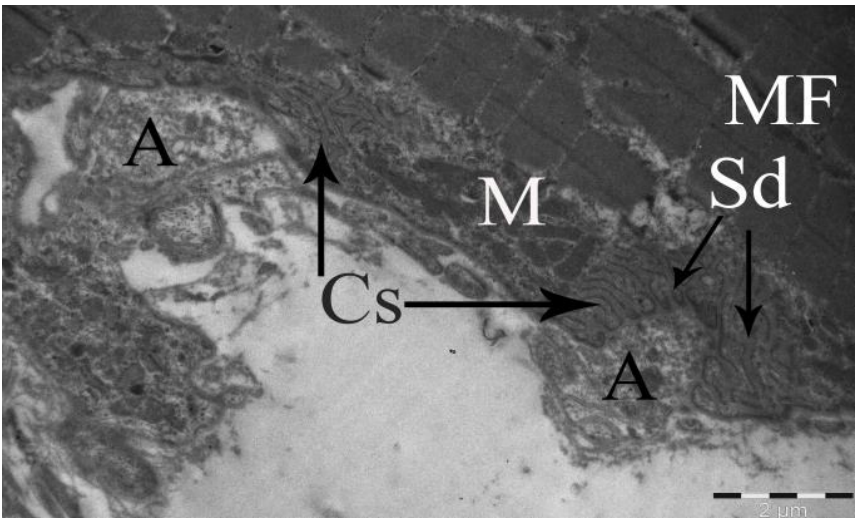

Figure 3a. Ultrastructure of denervated buccinators in rabbits of Treatment Group 2 after surgery using allogeneic biomaterials. The area of neuromuscular connections (motor end plates) on the surface of the fiber in denervated buccinator on day 180; MF - striated muscle fiber; $\mathrm{A}$ - the end of the motor nerve axon; M - mitochondria; Cf (-) - contact folds; Sc (-) - synaptic clefts. Magnification x8000

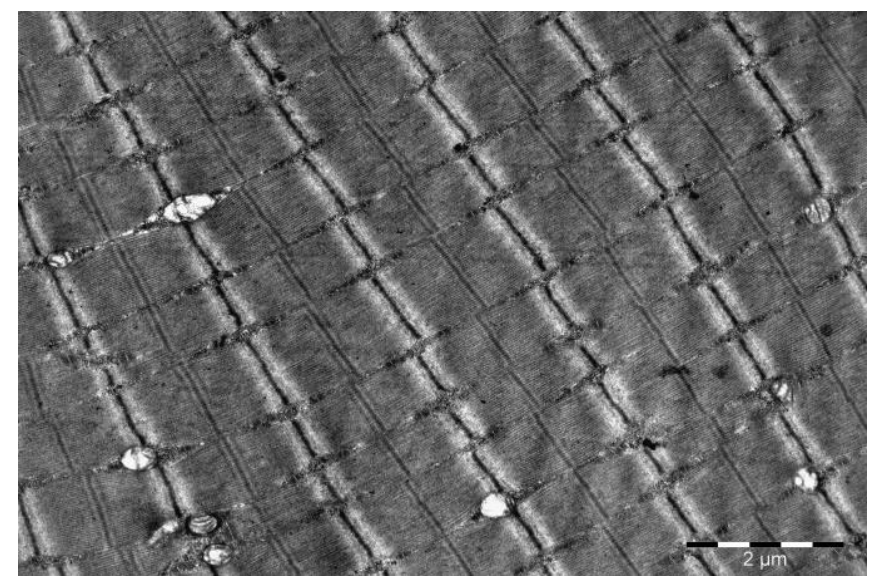

Figure $3 b$. Ultrastructure of denervated buccinators in rabbits of Treatment Group 2 after surgery using allogeneic biomaterials. Striated muscle fiber of the rabbit buccal muscle on day 180 after the operation. Magnification x5000 
In Treatment Group 2, the use of dispersed forms of allogeneic biomaterials along with an autograft of the masseter promoted the formation of a loose connective tissue area, more favorable for the sprouting of blood vessels and axons from the bundles of the trigeminal nerve on the autograft to the denervated buccinator. This fact was evidenced by the restoration of the fiber structure in striated musculature of the buccinator, as well as by the emergence of blood vessels in spaces between the muscle fiber bundles, and of neuromuscular junctions in the form of characteristic synapses on the surface of regenerating muscle fibers.

The results of multiannual research conducted at the Russian National Center for Eye and Plastic Surgery (Ufa) have shown that, when implanted into the tissues of a living organism, allogeneic biomaterials have the property of inhibiting cicatricial transformations. They are gradually resorbed by macrophages and replaced by newly formed tissue with full differentiation of its constituent elements [6, 7]. Nonspecific stimulation of macrophages with allogeneic biomaterial helps restoring the cellular mechanisms (disrupted by some pathology) of controlling tissue regeneration, which leads to reparative processes proceeding on the basis of physiological regeneration. Therefore, use of allogeneic biomaterials in surgical practice allows achieving complete repair and avoiding tissue scarring, including the muscle tissue.

The positive effect of allogeneic biomaterials on regeneration of muscle tissues and their innervation apparatus is also substantiated by the results of studies by D.A. Shcherbakov [8] and A.I. Lebedeva [9], reflecting the morphogenetic patterns in the field of transplantation with the participation of integrative systems in human body. The presented data on the muscle tissue reinnervation are used in the development of reconstructive surgeries on the auxiliary apparatus of the eyeball (eyelids, extraocular muscles). In particular, in the study of A.B. Nuraeva [10], based on using reinnervation of mimetic muscles during allotransplantation, the possibility of anatomical, and also of functional, restoration of the eyelids was proved. The comprehensive experimental and clinical study on transplant reconstructive surgeries performed on the soft frame of the orbit, including the musculature of the eyelids, presented a wide range of intersystem regulatory influences that ultimately implement reparative regeneration of highly differentiated anatomical structures. The data on the interaction of the allograft with the integrative systems of the body (lymphatic and immune) were also published. Rehabilitation technology of pharmacopuncture using Alloplant $\AA$ biomaterials, developed by E.R. Muldashev [7] and introduced at a number of clinics, could be considered from a standpoint of interacting systems of a human body.

The results of our study, as well as of other research projects, demonstrated a general pattern: the formation of morphofunctional connections between the center and the periphery with the restoration of homeostasis in the dynamics of replacement regeneration. It is extremely important that, in restoration of structural and functional correlations, a significant place is occupied by the transplantation of biomaterials, which is a factor of mobilizing both local and systemic reactions of the organism. Structural and functional restoration of the innervation apparatus of muscles reflects the most important postulate of the functional system: the principle of consolidating central and peripheral structures into the dynamic unity. In our case, we should emphasize continuous interaction among the transplant and its recipient, including interaction through the structures of the nervous system in order to achieve a positive result for the entire organism. The presented data allow considering the transplantation of biomaterials a factor of systemic and structural interactions in conditions of the holistic system of a human body, optimizing the evolutionarily formed pathways of maintaining local homeostasis.

\section{Conclusion}

Using biomaterials of the Alloplant $\AA$ series in myoplastic surgeries with reinnervation promotes inhibition of cicatricial transformations of damaged muscles, as well as regeneration of muscle cells and elements of both circulatory and nervous systems, thereby contributing to the establishment of stable structural and functional relations in the body during regenerative interventions. The results of the experiment with reinnervation of mimetic muscles can be considered an example of restoring interlevel relations in human body after the use of allogeneic biomaterials.

\section{Conflict of interest}

The authors declare no conflicts of interest pertaining to the study. The study was performed within the framework of the Government procurement No. 056-00110-18-00 approved by the Ministry of Healthcare of the Russian Federation on December 26, 2017.

\section{References}

1. Filatov VP. Selected works. In four volumes. Kiev: Publishing House of the UkSSR Academy of Sciences 1961; 2: 447 p. [In Russian].

2. Babaeva AG. Regeneration: Facts and prospects. Moscow: RAMS Publishing House 2009; 336 p. [In Russian].

3. Kryzhanovsky GN et al. Dysregulatory Pathology. Moscow: Medicine 2002; 632 p. [In Russian].

4. Sarkisov DS, Perov YuL et al. Microscopy Technique: Manual. Moscow: Medicine 1996; 117-8. [In Russian].

5. Shekhter AB, Serov VV. Inflammation, adaptive regeneration and 'dysregeneration' (analysis of intercellular interactions). Archive of Pathology 1991; 53 (7): 7-14. [In Russian]. https://pubmed.ncbi.nlm.nih.gov/1741672/

6. Muslimov SA, Musina LA, Lebedeva AI et al. Morphological basis of using Alloplant $\AA$ biomaterials for tissue regeneration. Morphology 2008; (2): 92-3. [In Russian].

7. Muldashev ER et al. Regenerative Medicine: Alloplant $囚$. Ufa: Bashkortostan State Republican Publishing House 2014; 432 p. [In Russian].

8. Shcherbakov DA. Anatomical Rationale for Myotendoplasty via Using Connective Tissue Allografts: PhD thesis abstract. Ufa, 2012; 22 p. [In Russian].

9. Lebedeva AI, Muslimov SA, Vagapova VS et al. Morphological Aspects of Skeletal Muscle Tissue Regeneration Caused by Allogeneic Biomaterial. Practical Medicine 2019; 17 (1): 98-102. [In Russian].

10. Nuraeva AB. Reconstructive Surgery for Deformities, Dislocations and Colobomas of Eyelids Using Alloplant Biomaterials: DSc dissertation abstract. Moscow 2018; 48 p. [In Russian]. 
Authors:

Lyalya A. Musina - DSc, Principal Research Scientist, Division of Morphology, Federal Budgetary Institution Russian National Center of Eye and Plastic Surgery, Ministry of Healthcare of Russia, Ufa, Russia;

Olga R.Shangina - DSc, Professor, Deputy Director-General, Head of the Laboratory of Tissue Preservation, Principal Research Scientist, Federal Budgetary Institution Russian National Center of Eye and Plastic Surgery, Ministry of Healthcare of Russia, Ufa, Russia;

Rafik T. Nigmatullin - DSc, Professor, Scientific Consultant, Federal Budgetary Institution Russian National Center of Eye and Plastic Surgery, Ministry of Healthcare of Russia, Ufa, Russia;

Sagit A. Muslimov - DSc, Head of the Division of Morphology, Principal Research Scientist, Federal Budgetary Institution Russian National Center of Eye and Plastic Surgery, Ministry of Healthcare of Russia, Ufa, Russia. 